\title{
Epidemic acute haemorrhagic conjunctivitis in Lagos, Nigeria
}

\author{
T. E. McMOLI, ${ }^{1}$ A. N. BORDOH, ${ }^{1}$ G. M. R. MUNUBE, ${ }^{2}$ AND E. J. BELL ${ }^{3}$ \\ From the 'Ophthalmology Unit, College of Medicine, University of Lagos; the ${ }^{2}$ Virology Section WHO, \\ Lagos; and the ${ }^{3}$ Regional Virus Laboratory, Ruchill Hospital, Glasgow
}

SUMMARY Enterovirus 70 has recently emerged as a causative agent of epidemic acute haemorrhagic conjunctivitis (AHC). This paper is a report of the first association of enterovirus 70 with epidemic AHC in Nigeria. Despite numerous symptoms, including reduction in visual acuity, eventual recovery in 2 to 3 weeks with no functional loss was the rule except in 11 patients. Five of these patients ended up with superficial corneal scarring. Two had evisceration for unresolving panophthalmitis, while 4 went blind from ruptured corneal abscesses or ulcers. All the 11 patients had treated themselves or used traditional medications. None of the patients had signs of involvement of the central nervous system.

Owing to limited investigative facilities the agent causing epidemic acute haemorrhagic conjunctivitis (AHC) which hit Nigeria between September and December 1969 was not determined. Type 8 adenovirus was suspected, ${ }^{12}$ and bacterial studies were negative.

The occurrence of another clinically identical epidemic in 1981 started further investigations. We here report for the first time evidence of enterovirus $\mathbf{7 0}$ as a cause of epidemic AHC in Nigeria.

\section{Materials and methods}

The study involved 126 consecutive patients with AHC seen at the Eye Clinic of Lagos University Teaching Hospital between February 1981 and June 1981. Questions were asked about age, sex, occupation, residence, duration of illness, associated systemic symptoms, previous medications, number of other affected members in the household or compounds, and past medical history of similar disease known locally as 'A pollo.' (The first epidemic, which occurred in 1969, coincided in West Africa with the return of the Apollo XI astronauts from the moon; the epidemic was then causally linked with moon dust.) Each patient was given a complete ocular and general medical examination including palpation for enlarged lymph nodes.

Correspondence to Dr E. J. Bell, Regional Virus Laboratory, Ruchill Hospital, Glasgow G20 9NB.
Conjunctival swabs from 71 patients were taken for virus studies and put into Hanks-based transport medium. These were subsequently stored at temperatures ranging between $-20^{\circ} \mathrm{C}$ and $-70^{\circ} \mathrm{C}$. Separate conjunctival swabs were also taken for bacterial microscopy and culture. These were put into Stuart's transport (Oxoid) medium. The swabs were later inoculated on to 2 blood bases and one heated blood base (Oxoid) agar plates (chocolate). One blood agar was incubated anaerobically for 24 hours at $37^{\circ} \mathrm{C}$ using the anaerobic (Oxoid) jar. The second blood agar and chocolate agar plates were incubated for 24 to 48 hours in a candle extinction jar.

Unfortunately only 7 acute phase serum samples

Table 1 Preclinic medications used by AHC patients at Lagos University Teaching Hospital

\begin{tabular}{lrrl}
\hline Medication & Number & $\%$ & $\begin{array}{l}\% \text { with } \\
\text { complications }\end{array}$ \\
\hline Nil & 60 & $47 \cdot 6$ & Nil \\
Chloramphenical eye drops & 24 & $19 \cdot 0$ & Nil \\
Sofradex eye drops & 13 & $10 \cdot 3$ & $0 \cdot 8$ \\
Sugar water & 11 & $8 \cdot 7$ & $6 \cdot 3$ \\
Ice water & 7 & $5 \cdot 6$ & $\mathrm{Nil}$ \\
Mentholatum & 3 & $2 \cdot 4$ & $\mathrm{Nil}$ \\
Kerosene & 3 & $2 \cdot 4$ & $0 \cdot 8$ \\
Breast milk & 2 & $1 \cdot 6$ & $\mathrm{Nil}$ \\
Camphor & 2 & $1 \cdot 6$ & $0 \cdot 8$ \\
Dettol & 1 & $0 \cdot 8$ & $\mathrm{Nil}$ \\
Total & 126 & 100 & $8 \cdot 7$ \\
\hline
\end{tabular}


were collected from the patients, 2 of whom returned for second samples to be taken. In addition single sera were taken from 12 patients 3 months later. Between July and August 198110 paired and 12 single sera were taken to serve as controls from unaffected healthy people aged between 18 and 60 .

All the specimens were air freighted in cool boxes to Glasgow for virus studies in October 1981. Eye swabs were inoculated into cell culture systems for attempted virus isolation, and sera were titrated against enterovirus 70 by a neutralisation test and adenovirus by a complement fixation test.

\section{Results}

\section{CASES OF ACUTE HAEMORRHAGIC} CONJUNCTIVITIS

Altogether 126 patients with $\mathrm{AHC}$ were treated at the Eye Clinic of the Lagos University Teaching Hospital, and from these patients detailed information was obtained. Fiftynine $(47 \%)$ were males and $67(53 \%)$ were females. Patients in this study were mainly adults, the majority being in the 20-29-year age group. Four patients, 3 males and one female, aged 25 to 35 gave a positive past history of having been affected during the 1969 outbreak of similar conjunctivitis. Family history indicated that 123 relatives had suffered from similar conjunctivitis around the same time but had not bothered to seek medical consultation or treatment.

The symptoms were initially unilateral but rapidly became bilateral within 24 hours in $99 \%$ of cases. Among the commonest symptoms in order of frequency were increasing ocular foreign body sensations, lacrimation, redness and swelling of eyes, and moderate to severe periorbital pains markedly aggravated by eye movements. Other symptoms included ipsilateral headache becoming diffuse as the second eye became affected. Eleven patients had stiff necks but no signs of central nervous system involvement. General body pains were experienced by 2 patients who also had herpes labialis. In 6 cases there was associated catarrh with no other symptoms of respiratory tract involvement.

Table 1 shows medications used by patients before attending the Eye Clinic. The most commonly used were chloramphenicol and Sofradex (framycetin, dexamethasone, and gramicidin) eye drops, sugar water, and ice water. It should be noted that dangerous substances such as kerosene were used for eye instillations. The commonest sign at first presentation was mild to moderate diffuse bulbar subconjunctival haemorrhages, which in a few cases increased in intensity from localised patches and became confluent within 24 hours of the infection. They then remained static until they were finally reabsorbed in the third to fourth week of the infection. Mild papillary hyperplasia of the palpebral conjunctiva was more common in patients seen in the first 24 hours of the infection, after which it slowly regressed or was masked in a few cases by petechial palpebral subconjunctival haemorrhages.

Altogether $11(8.7 \%)$ out of the 126 patients studied developed complications, namely corneal ulcerations, ${ }^{3}$ corneal perforations, ${ }^{4}$ and panophthalmitis. ${ }^{2}$ The commonest feature among patients with complications was the use of noxious self medications prior to clinic consultations. For example, 4 of our patients presenting with spontaneous corneal perforations had used sugar water frequently. The most pathetic of these were a 26-year-old mother with bilateral perforations and her 7-year-old daughter with unilateral perforation. Two other patients, both males, eventually required evisceration for unresolving panophthalmitis.

Though about $70 \%$ of the patients complained of impaired vision at first presentation, subjective tests (Snellen) showed no diminution of visual acuity. Fluorescein staining was positive in 5 patients with associated superficial corneal ulcerations. Rose Bengal was not used because it was not available.

Weekly follow up of the patients was quite disappointing, as only 15 patients turned up at the first visit, 4 for the second, and none at all for the third. On the other hand all 6 patients with severe complications such as corneal perforations and panophthalmitis kept their weekly appointments. When recalled by special effort 6 weeks later, however, $111(88 \%)$ of the AHC patients turned up for re-evaluation. With the exception of those with severe corneal complications already referred to and 2 others with superficial corneal scarring the remaining patients (103 out of the 111 who returned) had recovered completely with no visual deficits.

\section{LABORATORY FINDINGS}

Bacterial cultures yielded Staphylococcus albus in 11 out of 71 conjunctival swabs, representing approximately $15 \%$ of the total swabs. The 60 remaining specimens yielded no bacterial growths. Conjunctival smears stained with Gram and Giemsa stains showed a preponderance of lymphocytes among cellular elements seen.

In the absence of cell culture facilities conjunctival swabs from 71 patients were inoculated into newborn mice for attempted virus isolation. The results were all negative. The same samples that had been stored at $-20^{\circ} \mathrm{C}$ to $-70^{\circ} \mathrm{C}$ were inoculated 6 months later into tissue culture systems at the Regional Virus Laboratory, Ruchill Hospital, Glasgow, Scotland. No virus was isolated.

Serological tests carried out in Scotland, however, 
yielded a clue as to the causative agent for the AHC. Of the 2 paired sera available for testing from AHC patients one showed a greater than 4-fold rise $(<16 / 256)$ to enterovirus 70 and the second pair showed static high titres of 256. Although Kono et al..$^{5}$ state that titres $\geqslant 16$ to enterovirus 70 are of diagnostic value for AHC infection, we adopted as our criterion of positivity titres of 128 or greater. Thus, of a further 12 patients from whom only single convalescent phase serum samples were available 3 months after infection 7 had titres $\geqslant 128$ to enterovirus 70 ( 3 with 128,2 with 256 , and 2 with 512 ). All of the 10 control subjects without clinical conjunctivitis and from whom paired sera were collected at 3-week intervals had titres less than 32 . Single sera from 11 additional control subjects not suspected of having had AHC were also tested. Four had titres (1 with 128,3 with 256) suggesting that these individuals had suffered from mild or subclinical enterovirus 70 infection. Adenovirus serological studies were essentially negative both in AHC and control subjects. Thus all serological evidence available suggested that enterovirus 70 was the possible aetiological agent responsible for the epidemic of acute haemorrhagic conjunctivitis in Lagos State between January and June 1981.

\section{Discussion}

During the period from January to June 1981 an unusual outbreak of a highly contagious conjunctivitis hit Lagos State of Nigeria and affected a wide range of age groups. As this period coincided with the time when government-employed doctors all over the Federation were taking industrial action, most of the cases went unreported. However, it was observed that children in most primary and secondary schools in the State were affected, and children played an important role in disseminating the infection. Information gathered by the Lagos State Epidemiological Unit showed that at least 5014 cases attended medical centres in the local government areas of the State for treatment, of whom $2235(45.6 \%)$ were males, $1947(38.8 \%)$ females, and sex was not recorded in $832(16 \cdot 6 \%)$.

At the Lagos University Teaching Hospital Eye Clinic, which catered for staff members and their families, 126 patients were treated for AHC. Eye swabs taken from some of these patients failed to yield a common bacterial pathogen in culture or virus in newborn mice. Lack of tissue culture facilities led to prolonged storage of the specimens at fluctuating temperatures while arrangements were being made to dispatch them to the Regional Virus Laboratory, Ruchill Hospital, Glasgow, for further investigation. Failure to isolate the virus in cell cultures was not surprising, since the specimens had been stored under unfavourable conditions.

Though a limited number of serum samples were available for serological testing, sufficient information was obtained to incriminate enterovirus 70 as a causative agent of the epidemic of AHC that swept through Lagos State in the first half of 1981.

Besides Nigeria epidemics of AHC occurred in 1981 in Zaire, India, Pakistan, United Arab Emirates, Brazil, the Caribbean Islands, Central America, and the Southern United States. ${ }^{4}$ Where adequate investigations have been carried out, enterovirus 70 has been found responsible..$^{367}$

Towards the end of 1981 and the beginning of 1982 outbreaks and cases of AHC were still being reported in several islands of the west Pacific including Fiji, Karibati, Nauru, Niue, Ponape, Samoa, Tonga, and New Zealand. ${ }^{8}$

The 1981 epidemic of AHC occurred almost 10 years after the last pandemic caused by enterovirus 70. Where the virus hides between epidemics remains a mystery. The 1969 and 1981 epidemics hit Lagos State during dry weather when dry, dusty winds blow south-westerly from the Sahara Desert dispersing dirt and infected droplets. The dust causes irritation of the eyes and possibly impairs the integrity of the conjunctival mucosa, thus permitting infection with an agent which would otherwise have been kept at bay.

One important point is worth noting. Whereas enterovirus 70 induced conjunctivitis has a very good prognosis, blindness can follow if mismanaged, for corneal perforations and panophthalmitis occurred in some patients after self medication or attendance at traditional healers' establishments. It is therefore recommended that, if a similar outbreak appears in future, patients should seek proper medical treatment and avoid self medication.

We are indebted to Dr F. Assaad of the Virus Unit, WHO, Geneva, for linking us up with Professor N. Grist of the Regional Virus Laboratory, Glasgow, Scotland. We are grateful to Professor Grist for his helpful suggestions while this work was in progress. Finally, we acknowledge with thanks the competent technical assistance in the serological investigations rendered by $\mathrm{Mr} \mathrm{R}$. A. McCartney of the Regional Virus Laboratory, Glasgow, and the initial bacterial studies, storage, and packing of the specimens by Mrs G. A. Macaulay, chief medical laboratory technologist of the College of Medicine, University of Lagos, Nigeria.

\section{References}

1 Akinsete EO. Epidemic conjunctivitis in Lagos, Nigeria. J Nigeria Med Assoc 1970; 1: 46-50.

2 Salami MYI. Pattern of epidemic conjunctivitis among school age population in Lagos. J Nigeria Med Assoc 1971; 1: 184-8.

3 Desmyter J, Colaert J, Maertens K, Muyembe T. Enterovirus 70 Conjunctivitis in Zaire, 1981 Versus 1972. Lancet 1981; ii: 1054-5. 
4 Anonymous. Editorial. Lancet 1982; i: 372-4.

5 Kono R, Miyamura K, Ogino T, et al. Antibody titres to enterovirus type 70 in the 1981 Indian epidemic of acute haemorrhagic conjunctivitis. Lancet 1981 ; ii: 924-5.

6 WHO Weekly Epidemiological Record 1981; 56: 254.
7 Anonymous. Isolation of enterovirus 70 from patient with acute hemorrhagic conjunctivitis-Key West, Florida. Centers for Disease Control, Morbidity and Mortality Weekly Report 1981; 30: 497.

8 WHO Weekly Epidemiological Record 1982; 57: 22. 